Artículo

\title{
Competitividad y eficiencia económica de los sistemas de producción de guayaba en Calvillo, Aguascalientes
}

\author{
Mercedes Borja-Bravo ${ }^{1 \S}$ \\ José Alberto García-Salazar ${ }^{2}$ \\ Venancio Cuevas-Reyes ${ }^{3}$ \\ Sergio Arellano Arciniega ${ }^{1}$ \\ Silvia Xochilt Almeraya Quintero ${ }^{2}$ \\ ${ }^{1}$ Campo Experimental Pabellón-INIFAP. Carretera Aguascalientes-Zacatecas, Pabellón de Arteaga, \\ Aguascalientes, México. CP. 2067. ${ }^{2}$ Posgrado en Socioeconomía, Estadística e Informática-Colegio de \\ Posgraduados-Campus Montecillo. Carretera México-Texcoco km 36.5, Montecillo, Texcoco, Estado de \\ México. CP. 56230. ${ }^{3}$ Campo Experimental Valle de México-Programa de Socioeconomía-INIFAP. \\ Carretera los Reyes-Texcoco km 13.5, Coatlinchán, Texcoco, Estado de México. CP. 56250. \\ ${ }^{\S}$ Autora para correspondencia: borja.mercedes@inifap.gob.mx.
}

\section{Resumen}

La producción de guayaba (Psidium guajava) en Calvillo, Aguascalientes, tiene importancia socioeconómica para la entidad y requiere la aplicación de políticas públicas eficientes para mantener o incentivar su crecimiento. El objetivo fue determinar la competitividad, efectos de política y la eficiencia económica de los sistemas de producción de guayaba en el municipio de Calvillo, Aguascalientes. La matriz de análisis de política (MAP) se aplicó a la información técnico-productiva que se obtuvo de 28 encuestas realizadas a productores, estos se clasificaron como tradicionales, intermedios y empresariales. El análisis de los resultados mostró que los tres sistemas de producción son rentables, pero la relación costo privado (RCP) para los productores intermedios tuvo rentabilidad mayor (1.73) que los empresariales (1.64) y los tradicionales (1.38). La relación de costo de los recursos internos (RCRI) fue 0.55 para los productores tradicionales, 0.45 para los intermedios y 0.49 para los empresariales, esto indicó que son económicamente eficientes y, por consiguiente, cuentan con ventaja comparativa. En los tres grupos de productores se evidenció la existencia de desprotección de las políticas económicas a los sistemas de producción en detrimento de su competitividad. La produccion de guayaba en Calvillo, Aguascalientes genera riqueza y contribuye al ahorro de divisas para el país, lo que la convierte en una alternativa productiva para la región.

Palabras clave: bienes consumibles, factores internos, impuestos, rentabilidad.

Recibido: agosto de 2019

Aceptado: octubre de 2019 


\section{Introducción}

La guayaba (Psidium guajava) es una de las frutas con gran aceptación en el sector agroalimentario de México, por su alto contenido en vitamina $\mathrm{C}$ y compuestos fenólicos. En el periodo de 2012 a 2015 la superficie sembrada de guayaba en el país fue de 21.15 mil hectáreas (ha), con una producción de 297.65 mil toneladas (t). Las regiones de mayor producción se ubicaron en los estados de Michoacán con $46.4 \%$ de la fruta a nivel nacional y tuvo un rendimiento de $14.75 \mathrm{t} \mathrm{ha}^{-1}$, las de Aguascalientes y Zacatecas aportaron 30.6 y $16.5 \%$ de la producción y mostraron rendimientos de 15.31 y $15.42 \mathrm{t} \mathrm{ha}^{-1}$, respectivamente (SIAP, 2015).

La producción de Aguascalientes se desarrolla en el municipio de Calvillo, como una de las regiones de mayor importancia en la producción comercial de guayaba. Datos del SIAP (2015), indican que de 2012 a 2015, ocupó 6256 ha en Aguascalientes, lo cual fue 29.6\% de la superficie nacional plantada. En este periodo se produjo en promedio 94.87 mil toneladas de guayaba con un valor comercial de 361.82 millones de pesos, equivalente al $27.9 \%$ del valor de la producción nacional de guayaba, y el $15.16 \%$ del valor de la producción del sector agrícola de la entidad.

De 2005 a 2015, la superficie plantada y producción de guayaba en Calvillo observaron una disminución promedio anual de $-0.86 \%$ y $-1.2 \%$, respectivamente. Esta situación denota la pérdida de dinamismo de la actividad productiva provocada por: a) reducción del nivel de mantos acuíferos que ha generado escasez y encarecimiento en la extracción del agua de riego; b) fenómenos naturales como heladas, granizadas y sequías; c) presencia de plagas y enfermedades; y d) falta de paquetes tecnológicos adecuados para el manejo agronómico de la guayaba (De Luna, 2003).

Aunado a lo anterior, Sangerman-Jarquín et al. (2013) señalaron que el encarecimiento de los costos de producción y la caída en los precios reales de la fruta han repercutido en la rentabilidad de los productores de guayaba. Para Borja et al. (2016a) los aspectos anteriores, sumados a la poca planeación en la asignación de recursos públicos y privados han provocado la pérdida de rentabilidad, competitividad y sustentabilidad de la actividad económica de la guayaba en la región productora. Esta problemática se hace notar con el abandono de huertas y la reconversión productiva por otras especies como nopal verdura, jitomate y pastos.

A pesar de la problemática anterior, por su tamaño, especialización, cantidad de empleos que genera y el número de productores que hacen esta actividad, la cadena de guayaba está posicionada como de mayor importancia socioeconómica (Borja et al., 2016b), lo cual justifica la búsqueda de oportunidades para mantener o incentivar la actividad productiva de la guayaba en Aguascalientes.

Para que los productores encuentren alternativas que les permitan incrementar las ganancias de su cultivo, es necesario realizar un análisis detallado sobre la rentabilidad y competitividad de los sistemas de producción en Calvillo; así como, determinar la eficiencia económica o ventaja competitiva de la actividad. Para ello, se debe partir de comprender que la competitividad es la capacidad dinámica que tiene una cadena agroalimentaria localizada espacialmente para mantener, ampliar y mejorar de manera continua y sostenida su participación en el mercado (Romero y Sepúlveda, 1999); mientras que, la ventaja comparativa se refiere a la eficiencia con la cual un país puede obtener ganancias en el comercio con la producción de bienes, y esto porque los precios relativos al interior del país, no son los mismos que regulan el valor relativo de los productos al momento de comercializarlos con otros países (Romo y Abdel, 2005). 
La MAP es una herramienta que permite medir la competitividad de los sistemas de producción de la guayaba en función de la rentabilidad privada y económica, además de evaluar la eficiencia economica o ventaja comparativa basandose en un comparativo de los costos privados y económicos, para medir los resultados de la apertura comercial en los productores y la economía del país (Barrera-Rodríguez et al., 2011). Este tipo de análisis se ha realizado para la producción de guayaba en Michoacán, México (Zamora y Kido, 2008), para los sistemas de producción de vainilla (Vanilla planifolia J.) en la región del Totonacapan, México (Barrera-Rodríguez et al., 2011); así como, en sistemas de producción pecuarios (Barrón-Aguilar et al., 2000; LaraCovarrubias et al., 2003; Rebollar-Rebollar et al., 2011).

Lo anterior, proporcionaría información de primera mano a los responsables de formular las políticas del subsector y con ello diseñar estrategias enfocadas a mantener o incrementar la competitividad de la actividad en la región, y relativas a la asignación de recursos. Por lo anterior y considerando la importancia de la guayaba en el estado, el presente trabajo tuvo como objetivo determinar la competitividad, efectos de política y la eficiencia económica de los sistemas de producción de guayaba en el municipio de Calvillo, Aguascalientes.

\section{Materiales y métodos}

La investigación se realizó en el municipio de Calvillo, Aguascalientes, que se localiza en la parte suroeste del estado entre $21^{\circ} 42^{\prime}$ '54.00' ' y $22^{\circ} 06^{\prime} 25.20^{\prime \prime}$ ' latitud norte y entre $102^{\circ} 31^{\prime} 26.40^{\prime \prime}$ y $102^{\circ} 52^{\prime} 30.00^{\prime}$ ' longitud oeste, a una altura promedio de $1630 \mathrm{msnm}$, con una superficie de 908.23 $\mathrm{km}^{2}$ y representa $16 \%$ del territorio estatal, en 2015 tenía 56 mil habitantes (INEGI, 2015).

En la investigación se usó la matriz de análisis de política (MAP) propuesto por Monke and Pearson (1989), con la cual se realizó un análisis detallado de la estructura de costos con precios privados y económicos. La MAP permite evaluar la rentabilidad para el productor y la eficiencia económica de los sistemas; el objetivo de la metodología es medir el impacto de políticas gubernamentales y distorsiones de mercado en la rentabilidad privada y eficiencia del uso de recursos.

La MAP es un producto de dos indicadores contables, uno sobre la rentabilidad que define la diferencia entre los ingresos y gastos y la otra es la medición de los efectos de las divergencias (políticas y fallas de mercado) considerada como la diferencia entre los parámetros observados y los que existirían si se eliminaran las divergencias (Cuadro 1).

Cuadro 1. La matriz de análisis de política (MAP).

\begin{tabular}{|c|c|c|c|c|}
\hline \multirow{2}{*}{ Concepto } & \multirow{2}{*}{ Ingreso } & \multicolumn{2}{|c|}{ Costos } & \multirow{2}{*}{ Ganancia } \\
\hline & & Bienes comerciables & Factores internos & \\
\hline Precios privados & $\mathrm{A}$ & B & $\mathrm{C}$ & $\mathrm{D}$ \\
\hline Precios sociales & $\mathrm{E}$ & $\mathrm{F}$ & $\mathrm{G}$ & $\mathrm{H}$ \\
\hline Divergencias & $\mathrm{I}$ & $\mathrm{J}$ & $\mathrm{K}$ & $\mathrm{L}$ \\
\hline
\end{tabular}

$\mathrm{A}=$ ingreso bruto a precios privados; $\mathrm{E}=$ ingreso bruto a precios privados económicos; $\mathrm{B}=\cos$ tos de producción de los insumos comerciables valuados a precios privados; $\mathrm{F}=$ costos de producción de los insumos comerciables valuados a precios económicos; $\mathrm{C}=$ costos de los factores internos a precios privados; $\mathrm{G}=\operatorname{costos}$ de los factores internos a precios económicos; $\mathrm{D}=\mathrm{A}-\mathrm{B}-\mathrm{C}$ es la ganancia a precios privados; $\mathrm{H}=\mathrm{E}-\mathrm{F}-\mathrm{G}$ es la ganancia a precios económicos; $\mathrm{I}=\mathrm{A}-\mathrm{E}, \mathrm{J}$ = $\mathrm{B}-\mathrm{F}$ y $\mathrm{K}=\mathrm{C}-\mathrm{G}$ miden las transferencias a través del producto de los insumos comerciables y de los factores de producción; L= I-J-K= D-H mide las transferencias netas. Información obtenida de Monke y Pearson (1989). 
A partir del análisis de la MAP se generó el indicador de la relación de costo privado (RCP) que mide la capacidad del sistema de producción para pagar los recursos domésticos y el retorno al capital que representa la utilidad del productor (Barrón-Aguilar et al., 2000) y fue el indicador de competitividad, que resulta de dividir el costo de los factores de producción entre el valor agregado (diferencia entre el ingreso bruto y los costos de los insumos comerciables). Si RCP 1 significa que el productor es eficiente y tiene ganancia privada; si $\mathrm{RCP}=1$ no se generan ganancias y si RCP> 1, no habrá eficiencia privada (González y Alferes, 2010).

Para determinar el efecto de las políticas económicas se utilizaron los siguientes indicadores: El coeficiente de protección nominal (CPN) que es el cociente de la relación entre el precio del producto a precios privados entre el precio a precios económicos. Este indicador analiza las políticas de precios y permite saber si estos incentivan la producción interna; establece el grado de protección que tiene la producción debido a las políticas.

El coeficiente de protección nominal de los insumos (CPNI) mide el grado de transferencia en los insumos comerciales por las políticas comerciales y el tipo de cambio a través de los precios (González y Alferes, 2010); se calcula mediante el cociente del costo de los insumos comerciables a precios privados y su correspondiente a precios económicos. Si CPNI> 1 existe un subsidio y si CPNI 1 existe un impuesto implícito al precio interno de los insumos.

El coeficiente de protección efectiva (CPE) expresa el efecto combinado de las transferencias y los insumos comerciables derivado de las políticas comerciales y del tipo de cambio y se estima al dividir el valor agregado a precios privados entre precios económicos.

El equivalente de subsidio al productor (ESP) mide el nivel de transferencia neta desde o hacia los productores en su relación con otros sectores de la economía (L). El valor positivo del ESP indica una transferencia global de la sociedad al productor; mientras que, un valor negativo significa la transferencia global del productor a la sociedad y los contribuyentes (Adeoye and Oni, 2014).

La relación de costos de los recursos internos (RCRI) es el cociente de dividir el costo de los factores internos (G) valuados a precios de eficiencia (sin distorsiones) y el valor agregado a precios sociales (Monke and Pearson, 1989). Si RCRI< 1 el valor de los recursos internos usados en la producción es menor al valor de las divisas ahorradas, por lo que el país es eficiente económicamente en la producción de guayaba, caso contrario si RCRI> 1 el país es ineficiente en la producción (González y Alferes, 2010).

Para elaborar la MAP se recopiló información sobre coeficientes técnicos (rendimiento, y requerimientos de insumos) y precios a través de 28 encuestas aplicadas a productores de guayaba en el municipio de Calvillo, durante octubre y noviembre de 2015. Los productores encuestados fueron aquellos que tuvieron la disposición de brindar información detallada sobre registros de costos, cosechas y venta de fruta. A partir, de la información recopilada en las encuestas, se clasificaron para los productores en tres grupos tomando como referencia las características determinadas por Borja et al. (2018) (Cuadro 2). 
Cuadro 2. Clasificación de productores de guayaba en Calvillo, Aguascalientes.

\begin{tabular}{|c|c|c|}
\hline $\begin{array}{l}\text { Tipo de } \\
\text { productor }\end{array}$ & Características & $\begin{array}{c}(\%) \text { de } \\
\text { encuestados }\end{array}$ \\
\hline Tradicional & $\begin{array}{l}\text { Superficie plantada no mayor a } 2.5 \text { ha } \\
\text { Margen de producción de fruta de alta calidad }{ }^{\mathbb{I}} \text { entre } 30 \\
\text { y } 60 \% \text {. } \\
\text { No exportan }\end{array}$ & 36 \\
\hline Intermedio & $\begin{array}{l}\text { Superficie plantada mayor a } 2.5 \text { y menor a } 9 \text { ha } \\
\text { Margen de producción de fruta de alta calidad }{ }^{\mathbb{I I}} \text { entre } 39 \\
\text { y } 86 \% \text {. } \\
\text { Bajo volumen de exportación }\end{array}$ & 43 \\
\hline Empresarial & $\begin{array}{l}\text { Superficie plantada mayor a } 9 \text { ha } \\
\text { Margen de producción de fruta de alta calidad }{ }^{\mathbb{I}} \text { entre } 41 \\
\text { y } 79 \% \text {. } \\
\text { Mayor volumen de exportación }\end{array}$ & 21 \\
\hline
\end{tabular}

Información obtenida de Borja et al. (2015). "I= se refiere a la fruta de tamaño extra y primera, cuya clasificación se basa en la norma NMX-FF-040-SCFI-2002 (SE, 2002).

Se identificaron para cada productor los coeficientes técnicos de los bienes comerciables: fertilizantes químicos y orgánicos, plaguicidas, nematicidas, herbicidas, fungicidas, control biológico de plagas y enfermedades, herramientas agrícolas, combustible (ocupado en el traslado de personal a huertas, de producción y para el funcionamiento de equipo), recipientes y cajas para corte y los factores de la producción: asistencia técnica, jornales, certificaciones de la huerta, cuotas de pago de agua (que incluyeron la cuota por agua y el consumo de electricidad para el bombeo de agua), sistema de riego, equipos agrícolas (fumigadora manual y de motor, desbrozadora y seleccionadora), vehículo, bodegas, cercas, irradiación de la fruta y servicios administrativos.

Los precios de mercado de los bienes comerciables y equipo provinieron de la encuesta y de consultas hechas a las empresas Agricenter SA de CV; Agrícolas Guayaberos, SA de CV; Servi Agro de Calvillo y Ferquiagro, SA de CV y Tractores de Aguascalientes, SA de CV, el precio del jornal y costo del agua de riego los proporcionaron los productores. En la encuesta se preguntó sobre la cantidad y precio de la mano de obra familiar y contratada; la información fue utilizada para estimar el total del costo por mano de obra.

El costo de los bienes de capital se determinó como el costo de recuperación del capital considerando su vida útil, $20 \%$ del costo de adquisición como valor de rescate a una tasa de interés de nominal de $3.34 \%$ que fue la tasa de interés interbancaria de equilibrio (TIIE) promedio a 91 días para 2015 (BANXICO, 2015). En el análisis no se incluyó la renta de la huerta, ya que solo $7 \%$ de los productores lo realizaban; esta actividad es poco común al tratarse de un cultivo perenne que requiere inversión y las ganancias se reflejan en el largo plazo.

Con base a la metodología propuesta por Salcedo (2007), se calcularon los precios de paridad de importación principalmente para fertilizantes (sulfato de amonio, nitrato de calcio, nitrato de potasio, superfosfato y triple 17), plaguicidas, herbicidas, fungicidas y equipo agrícola. Los precios de paridad de importación se calcularon con los precios Free On Board (FOB) reportados por la Comisión Internacional de Comercio de Estados Unidos de América (USITC, 2015) y el Sistema 
de Información Comercial Vía Internet (SIAVI) (SE, 2017), después se calculó el precio CIF (Cost, Insurance and Freight), donde se consideró el costo de transporte marítimo de los fertilizantes que se obtuvo de cotizaciones realizadas a la empresa de transporte marítimo internacional Containers con puntos de salida en Florida y Miami y punto de llegada al puerto de Veracruz; también se calculó el costo de transporte ferroviario desde Veracruz a Aguascalientes, para ello se consultaron las tarifas y distancias de ferrocarril mexicano, SA de CV (FERROMEX).

En el caso del equipo y sistema de riego se estimó el costo por recuperación a precios de mercado y económicos. Para la conversión a moneda nacional se utilizó el tipo de cambio de equilibrio (TCE) que fue calculado para el periodo 2010 a 2015, tomando como año base 2013, el TCE se estimó con el método de la paridad del poder de compra (PPP) (Lara-Covarrubias et al., 2003), para lo cual se empleó el tipo de cambio nominal (BANXICO, 2015) y el índice de precios al consumidor de México y Estados Unidos de América (INEGI, 2016).

El tipo de cambio de equilibrio fue de \$13.16 USD, con un margen de sobrevaluación de $3.77 \%$ de enero de 2010 a diciembre de 2015. El precio económico de la guayaba se estimó con el precio de paridad de exportación (Salcedo, 2007), para ello se partió del precio CIF reportados por la Comisión Internacional de Comercio de Estados Unidos (USITC, 2015) colocado en McAllen Texas, USA, a este se descontó el costo de transporte, seguro y costos generados por la irradiación de la fruta y transporte local de las huertas a los empaques, la información fue proporcionada por las empresas empacadoras de guayaba que se localizan en el municipio de Calvillo y que son las que se encargan de la comercialización de la fruta para el mercado estadounidense.

Al costo del pago del riego se incrementó el subsidio otorgado por la Secretaría de Agricultura, Ganadería, Desarrollo Rural, Pesca y Alimentación (SAGARPA) dentro del Programa especial de energía para el campo en materia de energía eléctrica de uso agrícola (SAGARPA, 2016).

Al contar con las matrices de coeficientes técnicos y precios, se calcularon los presupuestos privados y económicos de los productores de guayaba. Al ser la guayaba un cultivo perenne, se proyectaron los presupuestos privados y económicos a 25 años sobre plantaciones ya establecidas y el año base fue 2015. El periodo de evaluación se determinó al considerar la experiencia de los productores, quienes mencionan que los arboles con 25 años de edad aún mantienen su vigorosidad y alto rendimiento.

Posteriormente, se estimó el valor presente neto (VPN) para cada presupuesto; en la actualización de los presupuestos se utilizó la TIIE promedio a 91 días (3.34\%) y la tasa Libor a 3 meses $(0.32 \%)$ para 2015 (BANXICO, 2015). El análisis se hizo con la información correspondiente a una superficie de 1 ha de guayabos para cada tipo de productor.

\section{Resultados y discusión}

\section{Rentabilidad privada y ventajas competitivas}

Las diferencias entre los costos totales según las características de los productores son evidentes. Los productores empresariales mostraron tener mayores costos de producción que los tradicionales e intermedios (Cuadro 3), la diferencia se explica porque los empresariales utilizan una mayor 
cantidad de fertilizantes químicos y abonos orgánicos (composta), exportan su producto por lo que destinan una mayor inversión al control químico y biológico de plagas y enfermedades; además, tienen una mayor superficie de huerta y requieren de un mayordomo que coordine las actividades de la plantación, lo que eleva el costo de la mano de obra.

Cuadro 3. Indicadores de rentabilidad privada del cultivo de la guayaba según características del productor en Calvillo, Aguascalientes.

\begin{tabular}{lccc}
\hline \multirow{2}{*}{ Ventaja competitiva } & \multicolumn{3}{c}{ Productores } \\
\cline { 2 - 4 } & Tradicionales & Intermedios & Empresariales \\
\hline Ingreso bruto $\left(\$ \mathrm{ha}^{-1}\right)$ & 48744 & 59702 & 79378 \\
Costo total $\left(\$ \mathrm{ha}^{-1}\right)$ & 35196 & 34468 & 48303 \\
Ganancia neta $\left(\$ \mathrm{ha}^{-1}\right)$ & 13548 & 25234 & 31075 \\
Rentabilidad privada & 1.38 & 1.73 & 1.64 \\
Retorno nominal al capital $(\%)$ & 38.5 & 73.2 & 64.3 \\
Costo de factores domésticos $\left(\$ \mathrm{ha}^{-1}\right)$ & 17931 & 17035 & 32571 \\
Valor agregado neto $\left(\$ \mathrm{ha}^{-1}\right)$ & 31479 & 42269 & 63646 \\
Relación de costo privado $(\mathrm{RCP})$ & 0.57 & 0.4 & 0.51 \\
\hline
\end{tabular}

Elaborado con base en la información de campo.

Los productores tradicionales e intermedios observan costos de producción parecidos, debido a la similitud en el manejo de las plantaciones, los costos más elevados en bienes comerciables fueron en combustible (34.2 y $32.6 \%$, respectivamente), ya que este insumo se utiliza para los traslados de las poblaciones a la huerta de los productores y jornaleros, para el funcionamiento de equipo y el transporte de la producción de la huerta a los centros de venta.

La mano de obra mostró tener gran participación en la estructura de costos, para los productores tradicionales fue de $37.5 \%$ y para los intermedios de $29.3 \%$, este alto costo se explica por el uso intensivo de mano de obra en labores como podas, deshierbe y cosecha de la fruta. Los costos en insumos comerciables fueron del 49.1, 50.5 y 32,6\% para los tradicionales, intermedios y empresariales; y los factores de la producción fueron de 50.9, 49.4 y 67.4\%, respectivamente.

El ingreso de los productores está determinado por el volumen de producción que obtengan de los diferentes tamaños de guayaba ya que de esto depende el mercado al que se dirige la fruta y el precio de venta. La guayaba 'extra' se caracteriza por tener una calidad superior y mide de 5 a más centímetros $(\mathrm{cm})$ de diámetro ecuatorial (SE, 2002), se comercializa como fruta en fresco en el mercado nacional y su precio promedio en 2015 fue de $\$ 7.5$ por kilogramo $(\mathrm{kg})$.

La fruta de 'primera' mide entre 4 y $4.9 \mathrm{~cm}$ de diámetro (SE, 2002), tiene la característica de ser comercializada en el mercado nacional y de exportación, ya que es la que cumple con las características en tamaño que prefiere el mercado estadounidense. En 2015, el precio de venta de los productores en el mercado nacional osciló entre 5.6 y $3.3 \$ \mathrm{~kg}^{-1}$ y en el de exportación entre 10 a $14 \$ \mathrm{~kg}^{-1}$. La fruta de tamaño 'extra' y 'primera' es considerada como de buena calidad. Por último, la guayaba de 'segunda' o 'canica' mide menos de $4 \mathrm{~cm}$ de diámetro (SE, 2002), el mercado donde se comercializa es la industria elaboración de pulpa, dulces típicos y postres; el precio de venta en 2015 fluctuó entre 2.4 y $1.6 \$ \mathrm{~kg}^{-1}$. 
Los productores empresariales son los que obtuvieron mejores ingresos brutos, la diferencia en el ingreso por tipo de productor oscila entre los 11 y 31 mil pesos por ha. Según los resultados obtenidos en la encuesta, los productores intermedios obtuvieron $20 \%$ de producción de extra y $59 \%$ de primera; mientras que, los productores tradicionales obtuvieron 20 y $46 \%$ y los empresariales 20 y $57 \%$. Sin embargo, una diferencia notable entre los productores intermedios y los empresariales es que estos últimos son exportadores, por lo que en promedio $18 \%$ de la producción total, que representa $31.6 \%$ de la producción de primera es la que dirigen a este mercado donde el precio es más alto y obtienen mayor margen de ingreso y ganancia.

Los productores intermedios y tradicionales comercializan en el mercado nacional como fruta en fresco y para la industria de procesamiento. Como se observa en el Cuadro 3, los tres sistemas de producción son rentables, pero son los productores intermedios los de mayor rentabilidad (1.73), seguidos de los empresariales (1.64) y los tradicionales (1.38). Según González y Alferes (2010) la rentabilidad privada es una condición necesaria pero no suficiente para determinar la competitividad privada, por tal motivo se deben analizar los demás indicadores.

El valor agregado neto indicó que después de liquidar los insumos comerciables de la producción hubo un remanente en el ingreso por la venta de una tonelada de guayaba. El valor agregado varió entre 64.6 y $80.2 \%$, estos valores significan que la producción de guayaba tiene una importante participación en la generación de empleos directos; es decir, que, de los costos de producción, el pago de mano obra representó 30.9\% y de acuerdo a lo expresado por Hernández-Martínez et al. (2008) el valor agregado neto es el monto del ingreso total utilizado en pagar la mano de obra, el pago del trabajo del productor y la ganancia que obtiene por la actividad; en el caso de la producción de guayaba en Calvillo, más del ingreso $60 \%$ se destina al pago de estos recursos.

Para los productores empresariales, la mano de obra es contratada y en los tradicionales e intermedios es mixta, ya que implica la ocupación de mano de obra familiar y contratada. Este indicador refleja el efecto de la producción al interior del sector agrícola (Morales-Hernández et $a l ., 2011)$. La competitividad se estimó con la RCP y en los sistemas de producción fue mayor a cero, pero menor a 1, lo cual indica que los tres sistemas fueron competitivos. Los sistemas de producción de guayaba mostraron tener capacidad para pagar los factores internos de la producción, además de obtener una ganancia por su actividad económica.

En el caso de los productores tradicionales, el costo de los factores internos representó el 57\% del valor agregado y $43 \%$ la utilidad, en los intermedios y empresariales los costos de los factores fueron 40.3 y $51.2 \%$ del total del valor agregado y la ganancia de 59.7 y $48.8 \%$. Estos resultados son similares a los reportados por Zamora y Kido (2008) para los sistemas de producción de guayaba en Michoacán, donde señalan que, bajo diferentes manejos agronómicos, el cultivo de la guayaba es eficiente al obtener un RCP menor a uno y ganancia privada positiva.

Con base en lo expresado por Rebollar-Rebollar et al. (2011), los sistemas de producción intermedia tuvieron una mayor competitividad y mayor grado de eficiencia privada, ya que son los que obtuvieron un RCP más cercano a cero; a menor RCP se incrementa la ganancia privada. 


\section{Efectos de la política económica en la competitividad}

El efecto de la política económica sobre la competitividad es la diferencia entre el presupuesto privado y económico, que refleja las distorsiones de política, subsidios e impuestos o la existencia de mercados imperfectos (Monke and Pearson, 1989). El CPNI en los sistemas de producción de guayaba se observó que para fertilizantes, fungicidas y herbicidas fue menor a la unidad, indica que los precios de mercado fueron menores que los precios económicos, por lo tanto, existe un impuesto implícito al precio de insumos (Cuadro 4) que los productores pagan al momento de la compra.

Cuadro 4. Indicadores de protección de precios en la producción de guayaba en Calvillo Aguascalientes, México.

\begin{tabular}{cccc}
\hline \multirow{2}{*}{ Protección de precios } & \multicolumn{3}{c}{ Productores } \\
\cline { 2 - 4 } & Tradicionales & Intermedios & Empresariales \\
\hline CPN de la guayaba & 0.27 & 0.39 & 0.42 \\
CPN de los fertilizantes & 0.42 & 0.57 & 0.78 \\
CPN de los plaguicidas & 1.75 & 1.53 & 1.53 \\
CPN de los fungicidas & 0.63 & 0.63 & 0.94 \\
CPN de los herbicidas & 0.62 & 0.57 & 0.61 \\
CPN de las herramientas & 1.23 & 1.18 & 1.26 \\
CPN del combustible & 1.36 & 1.36 & 1.36 \\
Coeficiente de protección efectiva (CPE) & 0.62 & 0.64 & 0.61 \\
Transferencia total (\$ ha ${ }^{-1}$ ) & -38654 & -47627 & -70688 \\
Equivalente de subsidio al productor (ESP) & -0.79 & -0.8 & -0.89 \\
\hline
\end{tabular}

Elaborado con base en la información de campo.

Los plaguicidas, herramientas y combustible tuvieron un CPNI mayor a 1 y denota que el precio doméstico excede el precio social, por lo que estos insumos mostraron tener un subsidio; este resultado es congruente, ya que algunos productores reciben apoyos gubernamentales estatales y federales para la compra a menor precio de plaguicidas, combustible, herramientas y equipo.

En cuanto al CPN de la guayaba, los resultados indican que, en 2015, los ingresos a precios privados fueron menores que a precios económico, esto se debió a que el precio nacional de la fruta fue menor al precio de paridad; es decir, que, en un mercado sin distorsiones, los productores tradicionales obtendrían un precio mayor $73 \%$, los intermedios $61 \%$ y los empresariales 58\%, por lo tanto, la guayaba es un producto que está desprotegido.

La explicación del porque es menor el precio de la guayaba nacional con respecto al internacional se centra en los siguientes puntos: a) el exceso de oferta temporal de la fruta, principalmente en los meses de noviembre a enero, lo que genera la caída del precio de venta; b) la producción de un alto porcentaje de fruta de 'segunda', lo que provoca que sea dirigida a mercados donde el precio de venta es bajo, tal es el caso de la agroindustria para la elaboración de pulpas, dulces, mermeladas y postres, donde el precio en el mercado nacional es menor $57.5 \%$ con respecto al tamaño 'primera' y 78.1\% al tamaño 'extra'; y 3) debilidad en las políticas para permitir el acceso a nuevos mercados. 
Al respecto, Zamora y Kido (2008) señalaron que después de más de diez años de iniciadas las primeras exportaciones de guayaba, es una fruta que no se ha podido consolidar a nivel internacional y la principal causa han sido las restricciones no arancelarias de tipo fitosanitarias que impiden comercializar el producto y los productores dejan de recibir la ganancia correspondiente a los precios sociales.

En el Cuadro 4 se observan los valores del coeficiente de protección efectiva para los productores de guayaba. En los tres grupos el CPE fue menor a uno, lo que indica que existe una desprotección en las políticas económicas, según Barrera-Rodríguez et al. (2011) los productores podrían haber recibido mayores remuneraciones a capital y mano de obra si no existieran distorsiones de mercado y si recibieran precios internacionales.

En términos porcentuales, los productores tradicionales, intermedios y empresariales debieron recibir 38, 36 y 39\% más de valor agregado, respectivamente; está perdida de valor se debió a las transferencias negativas vía insumos comerciales y factores internos (Cuadro 4); una de las causas de este resultado fueron el incremento en el tipo de cambio nominal durante 2015, el cual se mantuvo en un promedio de $\$ 15.9$ por dólar durante ese año; es decir, 19.13\% mayor al tipo de cambio de equilibrio (BANXICO, 2015); además de la alta tasa de interés interna.

El ESP para los productores tradicionales e intermedios fue de -0.8 y -0.9 para los empresariales; el valor negativo del ESP indica que los productores están sujetos a un impuesto implícito en la producción de la fruta y que hay una disminución en los ingresos brutos, por efecto de las transferencias originadas por las distorsiones de política comercial del producto e insumos y por las imperfecciones del mercado de los factores internos. El valor de ESP de los tres sistemas de producción fue menor a uno e indica la existencia de transferencia de recursos de los productores hacia la sociedad.

Resultados similares fueron encontrados por Zamora y Kido (2008) para los sistemas de producción de guayaba en Michoacán, quienes también argumentaron que si se eliminaran las distorsiones de política los productores incrementarían su nivel de ganancia privada.

\section{Rentabilidad económica y ventaja comparativa}

La finalidad de estimar el presupuesto económico es para analizar la ventaja comparativa que tienen los sistemas de producción de guayaba y determinar si para el país es más económico importar la fruta o producirla internamente. Los datos revelaron que los costos de producción a precios económicos de los sistemas de guayaba incrementaron, comparados con los costos a precios privados, esto como un efecto de la presencia de subsidios en los rubros de luz para el bombeo de agua (SAGARPA, 2016), adquisición de algunos insumos comerciales (fertilizantes) y equipos (DOF, 2015).

En una economía sin distorsiones, los ingresos incrementarían debido a que el precio de venta internacional de la fruta es mayor al nacional, esto provoca que el valor agregado en términos económicos incremente en los tres sistemas de producción (Cuadro 5). 
Cuadro 5. Indicadores de eficiencia económica del cultivo de la guayaba según el tipo de productor en Aguascalientes, México.

\begin{tabular}{lccc}
\hline \multirow{2}{*}{ Ventaja comparativa } & \multicolumn{3}{c}{ Productores } \\
\cline { 2 - 4 } & Tradicionales & Intermedios & Empresariales \\
\hline Ingreso bruto $\left(\$ \mathrm{ha}^{-1}\right)$ & 72126 & 88698 & 124622 \\
Costo total de la producción $\left(\$ \mathrm{ha}^{-1}\right)$ & 49259 & 52128 & 71864 \\
Costo de los factores internos $\left(\$ \mathrm{ha}^{-1}\right)$ & 27693 & 29918 & 51522 \\
Rentabilidad económica & 1.46 & 1.7 & 1.73 \\
Valor agregado neto $\left(\$ \mathrm{ha}^{-1}\right)$ & 50561 & 66488 & 104280 \\
Ganancia neta $\left(\$ \mathrm{ha}^{-1}\right)$ & 22867 & 36571 & 52,758 \\
Retorno económico del capital $(\%)$ & 46.4 & 70.2 & 73.4 \\
Relación de costo de los recursos internos $(\mathrm{RCRI})$ & 0.55 & 0.45 & 0.49 \\
\hline
\end{tabular}

Elaborado con base en la información de campo.

Para determinar la ventaja comparativa de la producción de guayaba se estimó el indicador de relación de los costos de los recursos internos (RCRI). El indicador se ubicó en un valor entre cero y uno para los productores de guayaba lo que indica que los tres sistemas de producción son económicamente eficientes y por consiguiente cuentan con ventaja comparativa.

A los productores tradicionales les cuesta 55 centavos producir un peso de valor agregado, a los intermedios 45 centavos y a los empresariales 49 centavos; además, al país le conviene producir guayaba porque ahorra 45,55 y $51 \%$ de las divisas que emplearía si decidiera importar guayaba.

\section{Conclusiones}

A precios privados, los productores de guayaba en Calvillo, Aguascalientes, son rentables y tienen competitividad. En los tres sistemas, el valor agregado cubre los costos de los factores internos y hay un remanente de ganancia extraordinaria, pero los productores intermedios son más eficientes en la producción, seguidos de los empresariales y tradicionales. Los productores pueden mejorar la rentabilidad y competitividad de su sistema al disminuir costos en insumos comerciables, mediante un uso más eficiente y racional de fertilizantes y productos para el control de plagas y enfermedades; asimismo, los productores empresariales deben optimizar el uso de los factores internos como mano de obra.

A precios económicos, los productores son rentables y obtienen ganancias con respecto a las privadas, lo cual conlleva a que la producción de guayaba en Calvillo presente ventaja comparativa con el exterior y se determina que los recursos son bien empleados porque esta actividad genera riqueza y contribuye al ahorro de divisas para el país, lo que la convierte en una alternativa productiva para la región.

Los productores de guayaba son afectados por la política económica mediante transferencias negativas traducidas en impuestos implícitos sobre algunos insumos comerciables como fertilizantes, plaguicidas y fungicidas, los cuales no son compensados con los subsidios que reciben en la compra de otros insumos y en el rubro de factores internos. Existe una desprotección en los sistemas de producción de la guayaba en Calvillo, este efecto resulta de la sobrevaluación del tipo 
de cambio y su constante incremento a partir de 2015 y las altas tasas de interés presentes en la economía nacional con respecto a la tasa de interés internacional, factores que encarecen los precios de los insumos y equipos utilizados en la producción. Por lo tanto, es conveniente que la política macroeconómica sea manejada de manera congruente para contribuir a la rentabilidad y competitividad de los sistemas de producción de la guayaba y del sector agrícola en general.

\section{Agradecimientos}

Los autores agradecen a los técnicos del Comité Estatal de Sanidad Vegetal de Aguascalientes (CESVA) y al Sistema Producto Guayaba del estado de Aguascalientes por la colaboración brindada en la fase de campo de la investigación. Asimismo, al Fondo Mixto CONACYT-Gobierno del estado de Aguascalientes por el financiamiento para la realización del presente trabajo.

\section{Literatura citada}

Adeoye I. B. and Oni O. A. 2014. Competitiveness and effects of policies on plantain production systems in Southwestern Nigeria. Agris online papers in economics and informatics. 6(4):3-13.

BANXICO. 2015. Banco de México. Sistema de información económica: tasa y precios de referencia. http://www.banxico.org.mx/SieInternet/consultarDirectorioInternetAction.do? accion $=$ consultarCuadro\&idCuadro $=\mathrm{CI} 34 \&$ locale $=$ es .

Barrera-Rodríguez, A. I.; Jaramillo-Villanueva, J. L.; Escobedo-Garrido y Herrera-Cabrera, B. E. 2011. Rentabilidad y competitividad de los sistemas de producción de vainilla (Vanilla planifolia) en la región del Totonacapan, México. Agrociencia. 45(5):625-638.

Barrón-Aguilar, J. F.; García-Mata, R. J. S.; Mora-Flores, S.; López-Díaz, A.; Pro-Martínez y García-Sánchez, R. C. 2000. Competitividad y efectos de política económica en la producción de cerdo en pie en 13 granjas porcícolas en el estado de Michoacán. Agrociencia. 34(3):369-377.

Borja, B. M.; Ramos, J. L. G.; De Lira, K. V. R. y Vélez, A. I. 2016a. La red de valor de guayaba en Aguascalientes. Folleto técnico No. 71. Instituto Nacional de Investigaciones Forestales, Agrícolas y Pecuarias (INIFAP). Aguascalientes, México. 26 p.

Borja, B. M.; Rodríguez, G. L.; Osuna, E. S. C. y López, L. A. 2016b. Importancia económica y competitividad de las cadenas agropecuarias en Aguascalientes, México. Investigación y Ciencia. 24(69):5-12.

Borja, B. M.; Velez, I. A. y Ramos, G. J. L. 2018. Tipologías y diferenciación de productores de guayaba (Psidium guajava 1.) en Calvillo, Aguascalientes, México. Región y Sociedad. 30(71):1-22.

De Luna, A. 2003. La guayaba en Calvillo Aguascalientes y factores que limitan su producción. In: Memoria del primer simposio internacional de la guayaba. Padilla, J. S.; Reyes, M. L.; González, G. E. y Perales, C. M. A. (Eds.). Aguascalientes, México. Instituto Nacional de Investigaciones Forestales, Agrícolas y Pecuarias (INIFAP). 184-196 pp.

DOF. 2015. Diario Oficial de la Federación. Reglas de operación de los programas de la Secretaría de Agricultura, Ganadería, Desarrollo Rural, Pesca y Alimentación (SAGARPA). http://www.gob.mx/cms/uploads/attachment/file/44530/Reglas-Operacion-2016-sagarpa.pdf.

González, E. A. y Alferes, M. V. 2010. Competitividad y ventajas comparativas de la producción de maíz en México. Rev. Mex. Cienc. Agríc. 1(3):381-396. 
Hernández-Martínez, J.; Rebollar-Rebollar, S. R.; Rojo-Rubio, J. A.; García-Salazar, E.; GuzmánSoria, J. J.; Martínez- Tinajero y Díaz-Carreño, M. A. 2008. Rentabilidad privada de las granjas porcinas en el sur del Estado de México. Universidad y Ciencia Trópico Húmedo 24(2):117-124. http://cuentame.inegi.org.mx/monografias/informacion/ags/poblacion/ educacion $\cdot$ aspx ?tema $=$ me $\& e=01$.

INEGI. 2015. Instituto Nacional de Estadística, Geografía e Informática. Información por entidad.

INEGI. 2016. Instituto Nacional de Estadística, Geografía e Informática. Banco de Información Económica (BIE): indicadores internacionales. http://www.inegi.org.mx/sistemas/bie/.

Lara-Covarrubias, D.; Mora- Flores, J. S.; Martínez- Damián, M. A.; García- Delgado, G.; OmañaSilvestre, J. M. y Gallegos-Sánchez, J. 2003. Competitividad y ventajas comparativas de sistemas de producción de leche en el estado de Jalisco, México. Agrociencia. 37(1):85-94.

Monke, E. A. and Pearson, S. R. 1989. The policy analysis matrix for agricultural development. 201 p. http://www.cepal.org/sites/default/files/courses/files/03_3_pambook.pdf.

Morales-Hernández, J. L.; Hernández-Martínez, J.; Rebollar-Rebollar, S. y Guzmán-Soria, E. 2011. Costos de producción y competitividad del cultivo de papa en el Estado de México. Agron. Mesoam. 22(2):339-349.

Rebollar-Rebollar, A.; Hernández-Martínez, J.; Rebollar-Rebollar, S.; Guzmán-Soria, E.; GarcíaMartínez, A. y González-Razo, F. J. 2011. Competitividad y rentabilidad de bovinos en corral en el sur del Estado México. Trop. Subtrop. Agroecosys. 14(2):691-698.

Romero, R. y Sepúlveda, S. 1999. Territorio, agricultura y competitividad. Cuaderno técnico núm. 10. IICA. http://www.fao.org/tempref/GI/Reserved/FTP_FaoRlc/old/proyecto/139jpn/ document/3dctos/3\%20referen/2ot/tac.pdf.

Romo, M. D. y Abdel, M. G. 2005. Sobre el concepto de competitividad. Rev. Com. Ext. 55(5):200-2014.

SAGARPA. 2016. Secretaría de Agricultura, Ganadería, Desarrollo Rural, Pesca y Alimentación. Programa especial de energía para el campo en materia de energía eléctrica de uso agrícola. http://www.gob.mx/sagarpa/acciones-y-programas/programa-especial-de-energia-para-elcampo-en-materia-de-energia-electrica-de-uso-agricola.

Salcedo, B. S. 2007. Competitividad de la agricultura en América Latina y el Caribe. Organización de las Naciones Unidas para la Agricultura y Alimentación (FAO). Santiago de Chile. 98 p.

Sangerman-Jarquín, D. M.; Larqué, S. B. S.; Navarro, B. A.; Schwentesius, R. R.; Damián, H. M. A. y Cuevas, S. J. A. 2013. Producción de guayaba [Psidium guajava (L.) Burm.] en el Estado de México, México. Rev. Mex. Cienc. Agríc 4(7):1081-1093.

SE. 2002. Secretaría de Economía. NMX-FF-040-SCFI-2002 Productos alimenticios no industrializados para consumo humano-fruta fresca-guayaba (Psidium guajava L.). http://www.sagarpa.gob.mx/agronegocios/Lists/Instrumentos\%20tcnicos\%20normalizaci n\%20y\%20marcas\%20colecti/attachments/92/nmx_guayaba.pdf.

SE. 2017. Secretaría de Economía. Sistema de Información Comercial Vía Internet (SIAVI). http://www.economia-snci.gob.mx/.

SIAP. 2015. Sistema de Información Agroalimentaria y Pesquera. Producción agrícola: cierre de la producción agrícola por estado.

USITC. 2015. United States International Trade Commission. DataWeb (U.S. Imports/Exports Data). https://www.usitc.gov/.

Zamora, A. I. y Kido, C. A. 2008. Rentabilidad y ventaja comparativa de la produccion de guayaba en el estado de Michoacán, México. INCEPTUM. 4:133-127. 